\title{
A Conversation with Mary Lou Jepsen
}

rom Tunisia to Taiwan, Mary Lou Jepsen has circled

3 the the globe in her role as CTO of the OLPC (One Laptop Per Child) project. Founded by MIT Media Lab co-founder Nicholas Negroponte in 2005, OLPC builds inexpensive laptops designed for educating children in developing nations. Marvels of engineering, the machines have been designed to withstand some of the harshest climates and most power-starved regions on the planet.

To accomplish this, Jepsen and her team had to reinvent what a laptop could be. As Jepsen says, "You ask different questions and you get different answers." The resulting machine, named the $\mathrm{XO}$, is uniquely adapted to its purpose, combining super-low-power electronics, mesh networking, and a sunlight-readable screen, which Jepsen designed herself. Although still shy of the "\$100 laptop" goal envisioned in the beginning, the $\mathrm{XO}$ is still the most inexpensive laptop ever built.

This fall, Jepsen took a break from her busy schedule to speak with us about some of the design challenges she and her team faced when developing the XO.

Interviewing Jepsen is her husband, John Ryan, a partner at Monitor Group, a technology consulting firm based in Cambridge, Massachusetts.

JOHN RYAN You've acknowledged several times that few computer projects would have chosen as their leader somebody whose core skills really aren't in computer architecture and design—or software, for that matter-but in display. As it turns out, that was actually an essential choice. Why is that, and where did that lead?

MARY LOU JEPSEN It defies conventional wisdom to put a display expert in charge of a laptop architecture, but since the cost of the screen in a laptop is more than $\$ 100$, it was the main barrier of entry to building a low-cost laptop.

What I've found coming to this project is that people who design computers don't know a lot about displays, and in fact by starting with the display and designing the computer kind of backwards, rather than just slapping a display onto a motherboard, we can design a whole new architecture.

The architecture we've created is very powerful, not

\author{
What's behind \\ THAT FUNKY \\ GREEN MACHINE?
}

just for low-cost laptops but for high-end laptops as well.

JR What you're saying is this is not just a costreduced version of the

commercial laptop that the typical office worker uses. The fundamental architecture here differs from your average commercial laptop. What are the key differences?

MLJ If you look at what's been happening in computers for the past 40 years, it's been about more power, more megahertz, more MIPS. As a result, we've had huge applications and operating systems. Instead, at OLPC we focused on an entirely different kind of solution space. We focused on low power consumption, no hard drive,

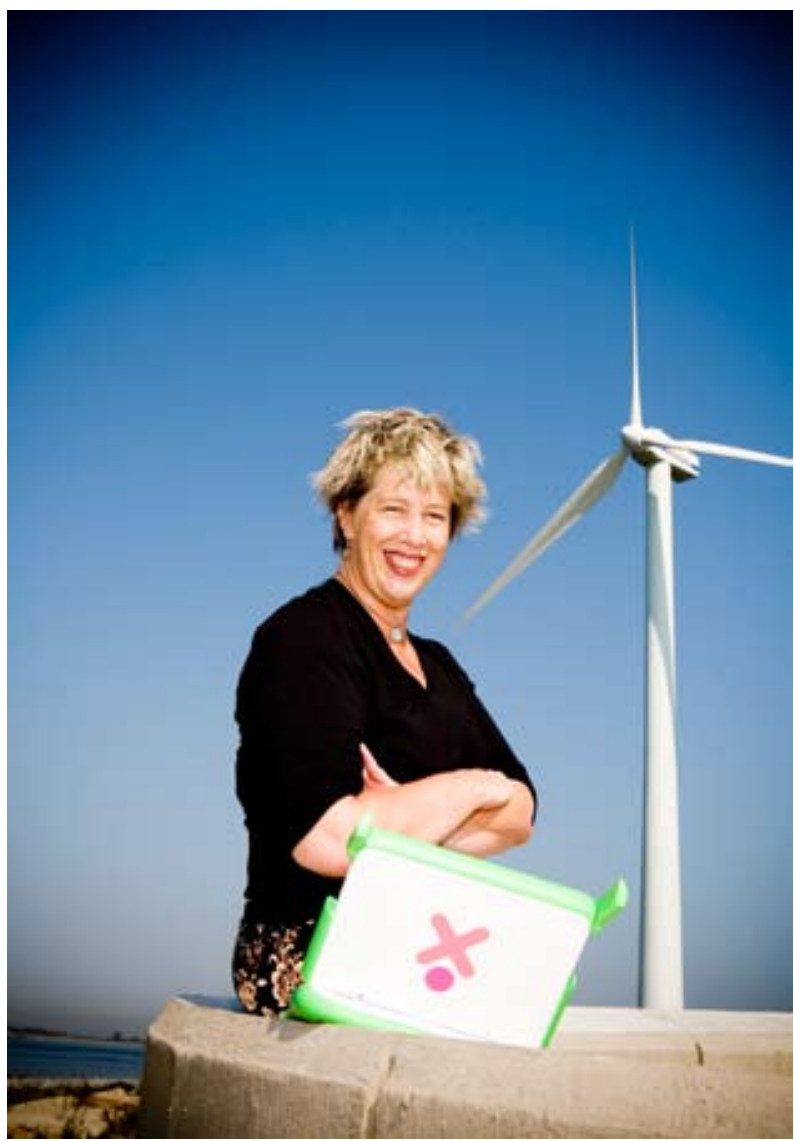


no moving parts, built-in networking, and sunlight-readable screens.

The whole machine is a different class of computing environment, and it's aimed at a different set of users. You can't get there by taking a classic office productivity laptop and cost-reducing it. You have to start by designing for the purpose and really looking at what those kids in Ghana or Peru or Pakistan need. You ask different questions and you get different answers.

JR Let's talk about the machines that are actually out in the field. How is this laptop's architecture relevant and appropriate to this use case?

MLJ It's pretty hot in much of the developing world, so we've designed a laptop that can take extreme heat. Part of that is an artifact of it being so low powered. We don't need big electrolytic capacitors whose lifetimes halve every 10 degrees hotter you get. We get to use little tiny capacitors because we've got so little power to deal with, and that's quite helpful.

Also, half the kids in the world don't have electricity at home. Half the kids. Eighty percent of the schools that we're going into don't have electricity. So we had to design a laptop that was also the infrastructure. It has mesh networking, which is the last mile, 10 miles, 100-mile Internet solution. The solar repeaters and active antennas that we've added into the mix cost about $\$ 10$ a piece and help to relay the Internet. If one laptop in a village is connected to the Internet, they all are.
Yes, it might be just a trickle, a low-bandwidth connection from the Internet to the laptop, but between the laptops is a high-bandwidth connection through the mesh network. We use 802.11s, which is the standard for mesh. The standard isn't actually complete, but we will be compatible with it when it's completed. We've had to make it up as we go along, so we're a little ahead of that. There's truly so little power in the developing world. If a

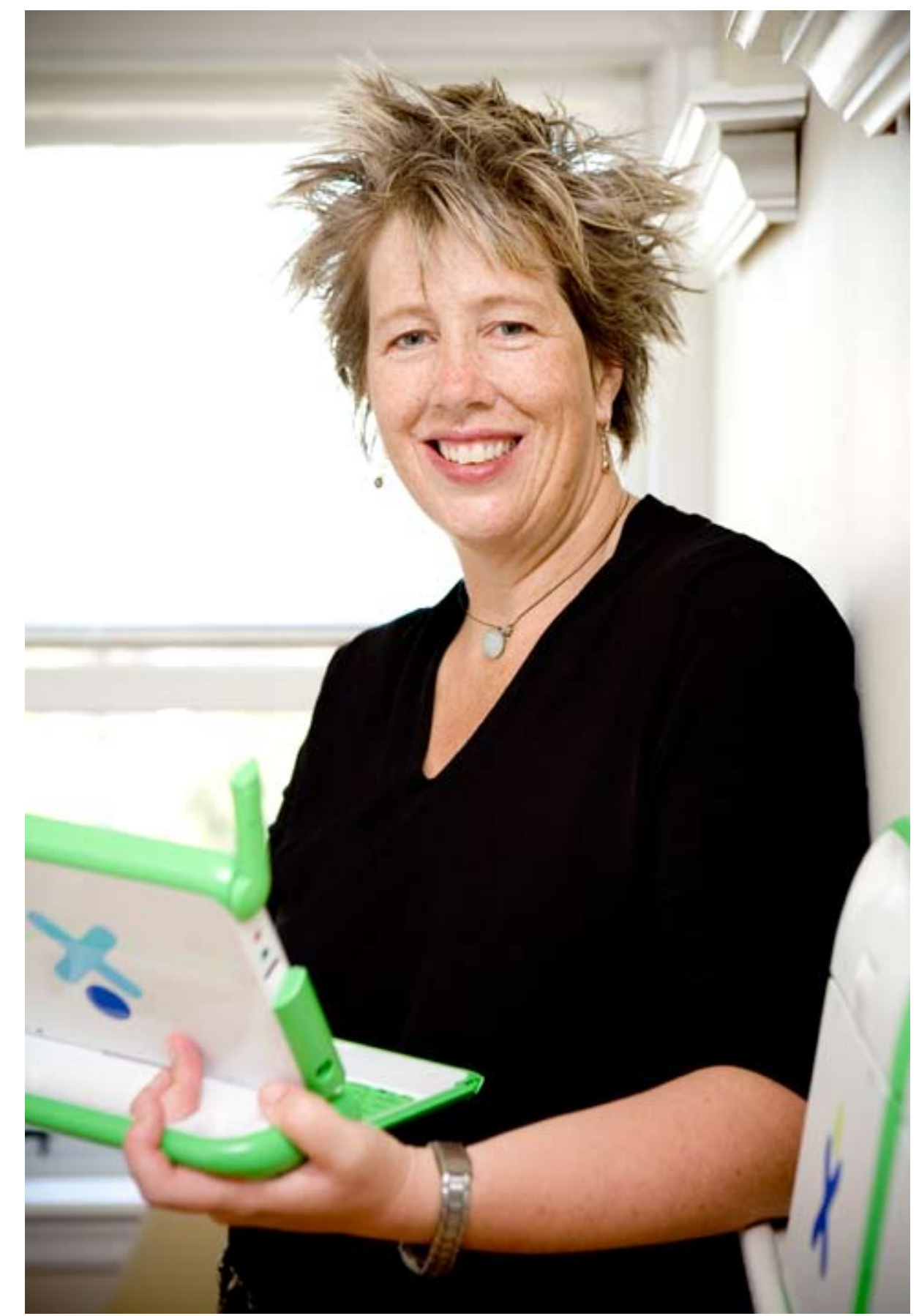


school is wired, it tends to be on a generator, and there's one 60-watt light bulb per classroom. Generators make really weird power. Usually what comes out of the wall in most countries is 50 or 60 hertz, or somewhere in between. With generators, the frequency of the AC power can go down to 35 hertz. We therefore had to do really interesting power conditioning on the AC adapter. The laptop itself can take between negative 32 volts to 40 volts, and work well with anything from 11 to 18 volts. You can plug a car battery into it. You can plug a solar panel into it. A hand crank can produce enough energy to power the battery for some time, as can a bicycle or a windmill. India has this cow-dung system that creates methane that drives a generator. Even that will work. JR There is one very important feature of the XO CPU, which is how fast you can turn it on and off. Maybe you can explain why that's essential.

MLJ It's almost the key feature. We use an AMD chipset, the LX-700, which allows you to turn the CPU on and off in a hundredth of a second. It's not noticeable to the users whether the motherboard is on or off because the moment they hit the keyboard or get a Skype phone call or what have you, the CPU and motherboard are back up and running.

That also allows us to run the mesh at extremely low power: 400 milliwatts, compared with my ThinkPad laptop, which uses approximately 10 watts just to run Wi-Fi.

Normally, the industry designs IT systems for the richest 1 billion people, but really the rest of the world doesn't need more gigahertz or 3D rendering. The key is having a laptop with batteries that last a long time-so that you can use it for a long time, outside and insideand being networked through the mesh. We're not designing the next supercomputer here. We're trying to make a machine that's low cost, low power, networked, and rugged so that people can use it in different environments, which are neither air conditioned with regulated power indoors nor well lit.

JR How did you bring the power consumption down?

MLJ We had to design a bunch of basics really quickly to make it work. We had to get rid of the hard disk, because not only is it the second most expensive component in a laptop after the screen, but it's also a huge power hog, and the number one cause of hardware failures is hard disk failure. That's three strikes against it.

Instead, we used flash memory, which people are starting to use. We had to fix a couple of bugs with the chipsets to make sure that they can talk to the flash memory, which operates at a very high speed. We had to do wear leveling on the flash. Luckily we've got David
Woodhouse, who wrote JFFS2 (Journaling Flash File System, version 2), on the project. He has been absolutely instrumental in every single build that we've done.

I should also talk about the low-power display. We did something I've been doing for a long time in different kinds of display technology: putting memory directly into the display itself. You can't do that with amorphous silicon, which is the standard transistor process used in LCD. To keep costs to a minimum, I used a standard process for the screen. But you can add memory in the timing controller. That means the screen can stay on while the rest of the motherboard or the chipset is off.

Why would you want to do that? It turns out that most of the time you're using a laptop or a desktop, the CPU isn't really doing much, even while it's running at multiple hundreds of megahertz. Right now I'm staring at my laptop. Not a single pixel on my screen is moving. What's the CPU doing on? What's the motherboard doing on? The way to get to low power-the big secret-is to turn stuff off that you're not using. But nobody has ever made a laptop with a screen that self-refreshes. You really do have to keep flipping the liquid crystal molecule; they like AC fields. The liquid crystal molecules fall apart in DC. You need to keep only two images in memory for that, and you can keep the screen on all day long.

We also put a tiny ARM core in our Wi-Fi chip. We used the Marvell chip because it's the only Wi-Fi chip with a tiny ARM core in it, which means Wi-Fi can also stay up and running while the CPU is off.

JR You've spoken about power consumption as critical, and about some of the consequences of this, such as suspend/resume, and the fact that this gives you more power options. Didn't that also have some impact on the battery? Didn't you have to make some choices about the battery, given that it was going to be used in hot environments and with kids?

MLJ There have been many battery recalls lately. Lithium ion is the dominant battery technology today in our digital portable equipment: every single laptop uses it, and a lot of cellphones use it, too. Nokia just recalled something like 46 million batteries, and, of course, we were very concerned. These are going to kids. We don't want exploding batteries.

In fact, we put the motherboard behind the screen so a kid can use the XO machine on his or her lap and it won't get too hot like a normal laptop. These are the first truly laptop-use computers made in the last decade.

We signed a memorandum of understanding with Libya about a year ago; each of the 1.2 million children there will get a laptop. It is the hottest place on earth. It 
gets to 57 degrees $\mathrm{C}$ in the desert in the summer, and the safe (read: don't explode) NiMH (nickel-metal hydride) batteries won't charge above 45 degrees $C$. That's a real problem because many spots in Libya are off the grid.

We started to look into other battery chemistries, such as lithium-ferro phosphate, which people haven't really used yet in consumer electronics. This chemistry charges in heat up to 60 degrees C. It's also about as safe as NiMH. We can put nickel-metal hydride or lithiumferro phosphate or, eventually, other battery chemistries into our laptops, which was another accomplishment. It was a real pain. We did that in the embedded controller. We also have a little fuel gauge in each battery that so we can keep track of its life cycle.

Our battery has a five-year life. You can go to 2,000 charge/recharge cycles. The lithium-ion battery in my ThinkPad is supposed to last for 500 charges, but in practice it's more like 200. So, moving to lithium-ferro phosphate is really cool because you don't have to spend additional money on periodic battery replacement costs, regardless of the environment.

Also, lithium-ferro phosphate is pretty environmentally friendly. Some early studies we did suggested that it possibly can decompose into fertilizer (with processing). Typically we think of batteries as environmentally bad, but there's some indication that lithium-ferro phosphate isn't that harmful. We haven't quite gone through all of the rigor on this, however, and it does require some processing to decompose it into fertilizer.

JR The XO machine is physically green. It has also been called "the greenest laptop on the planet," but that's not a reference to the color. What's that about?

MLJ It's the most environmentally friendly laptop ever made. It's also the lowest-cost laptop ever made.

The key to being environmentally friendly is to focus on power consumption. IEEE came up with the specification for this: IEEE 1680-2006. The EPEAT (Electronic Products Environmental Assessment Tool) system tracks how well laptops and desktops meet the IEEE spec. We just received an EPEAT Gold Award, the highest award, for our low environmental impact.

Although EPEAT and the IEEE standard mandate things like Energy Star compliance, the system doesn't note that we do 15 times better than Energy Star compliance; it doesn't even mention the lifetime of the laptop. Where a typical laptop has maybe a one-, two-, or maximum three-year lifetime in office workplaces, ours is double that at five years-and we do this in extreme environments to boot. Our machines are half the size and weight of a typical laptop, and our laptops are repairable by children and by locals. You can change out plastic parts quite easily, including the screens. Five-year-olds can change the screen on our laptop because it's actually that easy.

All that counts for environmental impact. We're trying to reduce the amount of e-waste in landfills. We're RoHS (Restriction of Hazardous Substances) compliant, plus-plus, which means we go beyond what the European Union has mandated as its reduction on hazardous substances. We have no mercury, no cadmium, no PCBs-a total reduction of hazardous substances.

We're also working on a take-back program, which is challenging in the developing world because there aren't roads and post offices in some places. We do have access to the schools, and we are working with them mostly on reuse. Rather than burning them down, it's better to cannibalize the machines and reuse the components.

JR How do you think computers will help education in the emerging world? You've built a software stack and GUI that are quite novel: How are these specific to the educational purpose of the machines?

MLJ The education situation in the developing world is so bad. School is basically $2 \frac{1}{2} 2$ hours a day from when the children are 6 until they're, let's say, 12, and then they go to work. They are sort of drilled and practiced to death. They learn how to sing, memorize, and exercise during their time. They get bored and drop out.

We wanted to design something that would engage kids beyond these rote-learning exercises, so the software architecture is completely different from a regular laptop. The user interface, called SUGAR, is based on the idea of everything being sharable; it explicitly enables collaboration. If you open a Web browser, if you open a write program, different kids can work with you. They can join in; they can share your activities.

To facilitate sharing, we have a mesh view in which each child is depicted by a color icon, which makes it easy to figure out where the other kids are on the network. The name of our laptop is $\mathrm{XO}$, so you can pick your color XO, and can see where your buddies are. You can write music with them. You can make videos with them. You can join in with them on anything.

JR There are fundamental elements of this machine that should ripple through general-purpose computing. When you talk to people and when you show them the laptop, what are their reactions? What are the features that make them say, "I want that in my machine"?

MLJ They want machines that are rugged, not fragile. 
They want long battery life-which means they need our power architecture. They want machines that are free of hazardous, toxic substances. They want better network connectivity. They want the screen-they want the screen desperately. It's not just kids who use the laptops under the trees in Africa. It's people who want to sit in outdoor cafes with their dogs and work on their laptops and blog in rich places. And, of course, it's useful for people who want to take computers into places that are a little too rough for today's expensive notebooks.

JR You designed the sunlight-readable screen. Can you tell us how?

MLJ I came up with this idea for a sunlight-readable screen by starting with a transflective process, which was used briefly on cellphones but wasn't very readable inside or outside (it was very dim) and so was dropped from most products. What I did differently was to put color filters over the transmissive part of the pixel only, instead of the mirror part of the pixel, and I usedget this-a colorless color filter over the rest of the pixel as a spacer.

In a transflective display, part of the pixel is reflective and part of it is transmissive. People think of it as kind of the worst of both worlds, so it hasn't been that popular. Again, these displays are considered dim, high power, and not that readable.

Each big LCD fab had developed a transflective process, so, I thought, why not use this process and then change the pixel layout by putting a little sliver of a color filter (red, green, or blue) over part of each pixel and then changing the bulk layout of the pixel as well? For best compatibility with minimal image-generation systems, I used diagonal stripes of color so that we would get square-root-of- 3 resolution in $\mathrm{X}$ and $\mathrm{Y}$ in color mode, when compared with the black-and-white (grayscale) resolution. You see, each pixel is black-andwhite (grayscale) in reflection and shows a single color in transmission (red, green, or blue when the backlight is on). Strictly speaking, therefore, you get one-third the

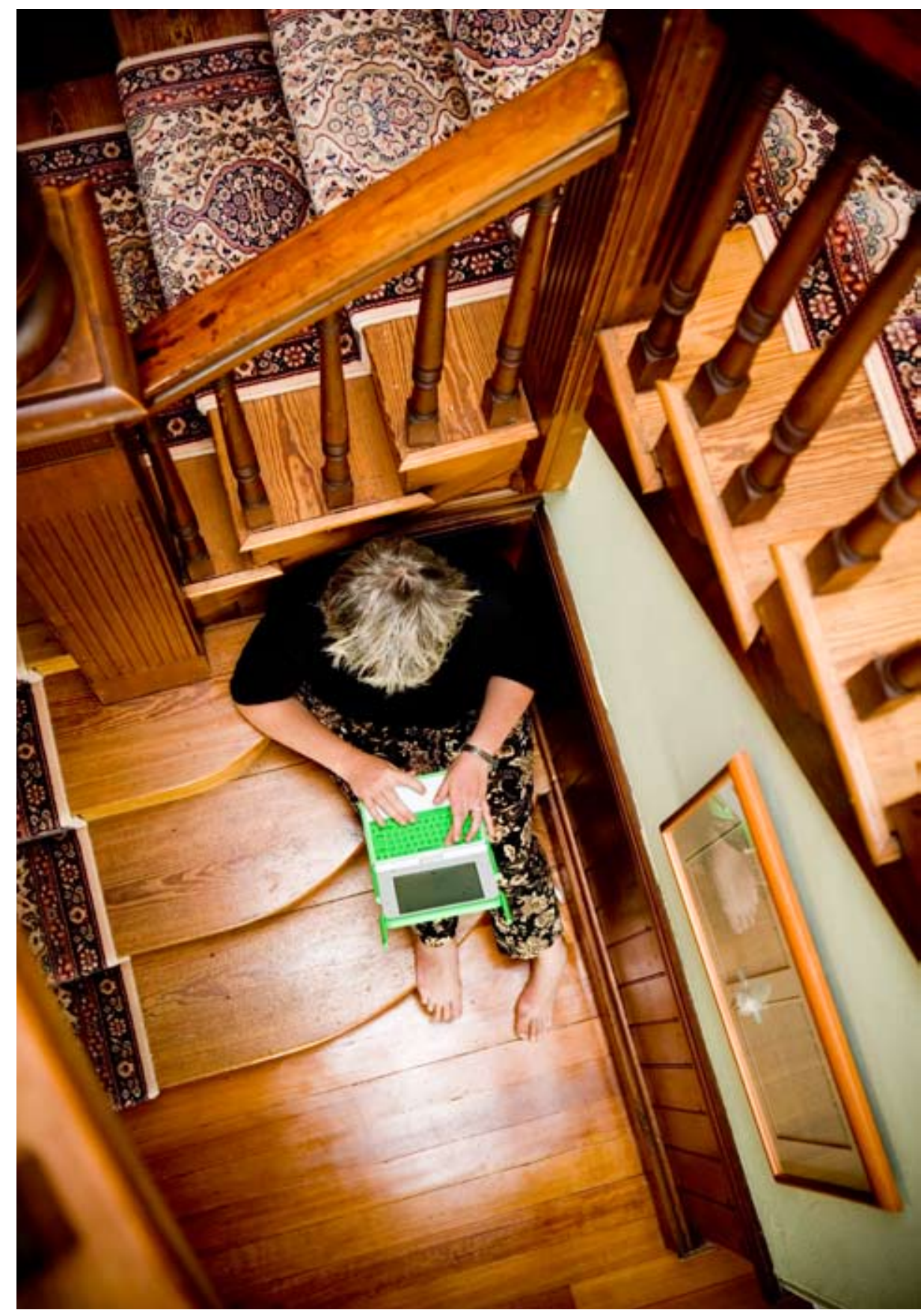


resolution in color. The human visual system isn't digital, however. It's analog and biological, and through this pixel layout we get higher perceived resolution-about 800 by 600 in color. You get all this with a low-cost and low-power TTL (transistor-transistor logic) interface that can be used because the true pixel count is so low. I'm just making the pixels do double or triple duty.

Since we decided to generate the full 1200-by-900 by $R, G$, and B for each pixel in the CPU chipset, however, I'm working on modifying the pixel layout to give even higher color resolution for the XO system next year.

The basic idea for this kind of thing is something that has been noted in encoding for a long time. NTSC (the dominant television standard in North America), for example, uses a much higher-luminance black-andwhite (grayscale) channel resolution, three or four times that for chrominance (color). A lot of that has to do with how NTSC had to be back when it came out, and the requirement for the transmission standard to be backwards compatible with black-and-white television signals. Subsequently, PAL (the dominant television standard in Europe) chose it, as did MPEG.

MPEG's luminance resolution is four times its chrominance resolution. In standard display making, however, we do exactly the opposite. Our color resolution is three times higher than the luminance resolution because it takes an R, G, and B subpixel to make a black-and-white pixel. Therefore, I gave part of the pixel a mirror for outdoor reading; and to make it brighter, I took the color filter off the mirror. Three-quarters of the light is absorbed by color filters, and I wanted the screen to have a bright e-paper mode. This way the luminance channel is three times the chrominance channel, and you get sunlight readability that makes the screen readable even in a bright room without the backlight on.

The screen is a good match to the way we see. To imagine the near future of display, we should probably take a look at what people in encoding have been doing for years and translate that into what it could mean in the display, since those people are just so clever in how they move bits around. The trick for display is to figure out a way to realize it in hardware. By the way, the display is also hardware, but hardware people don't usually think like that, in my experience.

Of course, there's also this thing that has been happening in display technology in the past 10 years called HDTV, and much of it is just madness and specsmanship (I worked in HD for seven years). The required contrast ratio for a display is now about 2,000 to 1, and the human visual system can't see more than 500 to 1 . It's nuts. 
I started to look at what has been happening in specsmanship and the perception of what quality is, rather than how the human visual system perceives quality. The disparity between the two is so great that there was a huge ability to cost down by lowering what would conventionally be called the "quality" but is actually something that we all can't really see.

My customers, poor kids in Africa, don't need HDTV. What they need is a low-cost laptop with a really good screen that makes e-books very readable.

Every minister of education I've talked to can never remember resolutions- 1,024 by 768 or 1,280 by 1,024 or whatever. So I made a new standard: 1,200 by 900 . It's a 71/2-inch diagonal, 6 - by $4 \frac{1}{2} 2$-inch screen, exactly 200 dots per inch. It's the size of a sheet of paper in the average-size book. It's super low power. There are LEDs in the backlight. It has no mercury.

JR Could we wrap up by hearing a few of the personal things that you've heard from some of the poorer kids who have already got their fingers on the laptop?

MLJ What really sticks in my mind is an e-mail I received from a kid named Badmus who lives just north of Abuja in Nigeria. He said he loves his laptop more than his life-and he's got a really tough life. There are a lot of kids like Badmus out there who could really use a new world, a new opportunity.

Think about these machines going into refugee camps. If you end up in a refugee camp, you have been through hell. It would be a really good thing if you could lose yourself in a laptop and start to find another world.

Some of the teacher feedback is very humble, thanking us so much for making the laptops, and telling us how they had been able to take the kids only so far, but now with the laptops, they're all learning together.

One of the teachers spends each evening at her school. She says there's nothing new to learn at home. This is very exciting. The kids stay at school in the evenings, too. I think there's new life in the schools we've gone into. If we have helped them by putting the power of computers and the Internet in front of them-if we've even made a step on that journey-this is much more than powerful machines delivering a gazillion MIPS. It has to be the most powerful thing we can do with computers. $Q$

\section{LOVE IT, HATE IT? LET US KNOW}

feedback@acmqueue.com or www.acmqueue.com/forums

() 2007 ACM 1542-7730/07/1100 $\$ 5.00$ 